\title{
AUTOMATIC CONTOUR ESTIMATION IN FETAL ULTRASOUND IMAGES
}

\author{
Sandra V. B. Jardim \\ Escola Superior de Tecnologia \\ Instituto Politécnico de Castelo Branco \\ 6000-767 Castelo Branco, Portugal \\ digiplan@netvisao.pt
}

\author{
Mário A. T. Figueiredo \\ Instituto de Telecomunicações \\ Instituto Superior Técnico \\ 1049-001 Lisboa, Portugal \\ mtf@lx.it.pt
}

\begin{abstract}
This paper describes a new method for automatic estimation of the contours of the femur and of the cranial cross-section in fetal ultrasound images. Our approach can be described as a regionbased maximum likelihood formulation of parametric deformable contours. This formulation provides robustness against the poor image quality, and allows simultaneous estimation of the contour parameters together with other parameters of the model. Implementation is carried out by a deterministic iterative algorithm with minimal user intervention. Experimental results testify for the very good performance of the approach.
\end{abstract}

\section{INTRODUCTION}

\subsection{Fetal Ultrasound Imaging}

Ultrasound imaging (echography) is a very important and competitive medical diagnostic tool, due to its low cost, short acquisition time, and non-invasive nature. This fact has stimulated a great amount of research aimed at increasing its diagnostic potential [6].

In obstetrics, measurements based on echographic images play a key role as a means for gestational age estimation. Several parameters are used as age and development indicators, the most important being biparietal diameter (BPD), occipital-frontal diameter (OFD), head circumference (HC), and femur length (FL) [14]. Each of these parameters provides, via a specific mathematical expression, estimates of the gestational age [14]. Consistency and reliability of these measurements is thus a crucial issue. Automatic techniques for the estimation of the contours of these anatomic structures can contribute to eliminate human variability.

Ultrasound images typically present several types of perturbations: displaying of non-structural echoes, removal of real structural echoes, displacement and distortion of echoes [14]. Moreover, being a coherent imaging technique, echography is characterized by the strong presence of speckle noise, which follows a Rayleigh distribution and can not be modelled as being independent nor additive [1]. All these perturbations make conventional contour estimation and segmentation techniques, usually based on local information, inappropriate for these images [5]. Although several special purpose schemes have been proposed for cardiac echography (see [5] and references therein), very few attempts have been made at automatizing the analysis of fetal images $[3,9]$.

This work was partially supported by the (Portuguese) Foundation for Science and Technology (FCT), grant POSI/33143/SRI/2000.

\subsection{Deformable Contours and Models}

Snakes, or active contour models [10], and their conceptual descendants, have been often adopted to deal with contour/boundary estimation problems in several medical imaging modalities [12]. Conventional snakes have several drawbacks (such as the strict use of local data) which have stimulated a great amount of research $[2,4,7,11,12,13,16]$. Although most limitations of the original formulation have been successfully addressed, only specialpurpose approaches have been able to deal with ultrasound images [5, 12]. Moreover, active contour models (or their probabilistic reformulations $[5,7,16])$ require careful tuning of several parameters, such as those controlling the trade-off between smoothness/robustness and estimation accuracy. This fact limits the applicability of these methods in practical medical imaging scenarios. Moreover, the quality of echographic images is often so low that the simple smoothness constraint used in snake-like approaches is not sufficient to ensure adequate contour estimates [12].

Parametric deformable models constitute another important approach to contour estimation which has been often used in medical image analysis (see [8] and references therein). Here, global shape models with a small number of parameters are generally used, in contrast with snakes, which typically use nonparametric contour descriptions. These model parameters are estimated in the presence of the observed image. In parametric models, it is possible to tailor the parameterization to the particular shape that is being fitted; this is usually known as a deformable template [15].

\subsection{Proposed Approach}

In this paper, we describe a new method for automatic estimation of femur and cranial cross-section contours in fetal echographic images. As mentioned above, this is the key step in an automatic measurement system. To deal with the low quality of ultrasound images, we describe the contour shapes using low order parametric deformable models. This low-order parameterization is sufficient to accommodate the expected shape and size variations, yet provides robustness against noise, image artifacts, and regions of missing data. The problem is formulated in a statistical estimation framework, and implementation is carried out by unsupervised deterministic iterative algorithms. 


\section{MAXIMUM LIKELIHOOD PARAMETRIC DEFORMABLE MODELS}

\subsection{Probabilistic Image Model}

Our approach consists of a maximum likelihood estimation approach to parametric deformable models. The basic building block is a probabilistic observation model $p(\mathbf{Z} \mid \theta)$ characterizing the observed data $\mathbf{Z}$ given the parameter vector $\theta$ which describes the contour shape. Under the maximum likelihood (ML) criterion, the best estimate of $\theta$, denoted $\widehat{\theta}_{\mathrm{ML}}$, is given by

$$
\widehat{\theta}_{\mathrm{ML}}=\arg \max _{\theta} p(\mathbf{Z} \mid \theta) \text {. }
$$

To derive the likelihood function $p(\mathbf{Z} \mid \theta)$, we adopt a regionbased approach; this has been shown to provide robustness with respect to local artifacts and poor image quality $[5,7,8,13,16]$. In our region-based model, $\mathbf{Z}$ consists of all the image data, thus being less sensitive to noise and image artifacts than methods that use local derived information (such as gradients or edges). In particular, we consider a simple model in which the image is divided into two regions, inside and outside, separated by the boundary to be estimated.

The observed image $\mathbf{Z}$ (an array of gray levels), is modelled as a random function of the object's boundary curve $\mathbf{v}(\theta)$, which is a function of the unknown parameters $\theta$. Moreover, $\mathbf{Z}$ may also depend on some additional observation parameters $\phi$. Accordingly, our likelihood function can be written as $p(\mathbf{Z} \mid \theta, \phi)$.

The simplest possible region-based model is characterized by the two following hypotheses: conditional independence (given the region boundary, all the pixels are independent); and region homogeneity (the probability distribution of each pixel only depends on whether is belongs to the inside or outside region). Thus, the likelihood function can be written as

$$
p(\mathbf{Z} \mid \theta, \phi)=\prod_{(i, j) \in I(\mathbf{v}(\theta))} p\left(Z_{(i, j)} \mid \phi_{\text {in }}\right) \prod_{(i, j) \in O(\mathbf{v}(\theta))} p\left(Z_{(i, j)} \mid \phi_{\text {out }}\right),
$$

with $Z_{(i, j)}$ denoting the value of pixel $(i, j)$, while $I(\mathbf{v}(\theta))$ and $O(\mathbf{v}(\theta))$ are, respectively, the inside and outside regions of the contour $\mathbf{v}(\theta)$. Finally, $p\left(Z_{(i, j)} \mid \phi_{\text {in }}\right)$ and $p\left(I_{(i, j)} \mid \phi_{\text {out }}\right)$ are the pixel-wise probability functions of these two regions.

Given that ultrasound images are well described by Rayleigh distributions, the pixel-wise probability densities have the form

$$
p(x \mid \phi)=\frac{x}{\phi} \exp \left\{-\frac{x^{2}}{2 \phi}\right\}, \text { for } x \geq 0,
$$

and thus $\phi=\left[\phi_{\text {in }} \phi_{\text {out }}\right]$, where $\phi_{\text {in }}$ and $\phi_{\text {out }}$ are the variances for the inside and outside regions, respectively.

\subsection{Complete Estimation Criterion and Algorithm}

To obtain an unsupervised scheme, we must estimate, from an observed image $\mathbf{Z}$, not only the parameters that define the contour, $\theta$, but also the other parameters $\phi$. Accordingly, we extend the maximum likelihood criterion to include also these parameters:

$$
(\hat{\theta}, \hat{\phi})=\underset{\theta, \phi}{\arg \max }\{\log p(\mathbf{Z} \mid \theta, \phi)\}
$$

Since solving (4) simultaneously with respect to $\theta$ and $\phi$ would be computationally very difficult, we settle for a suboptimal solution

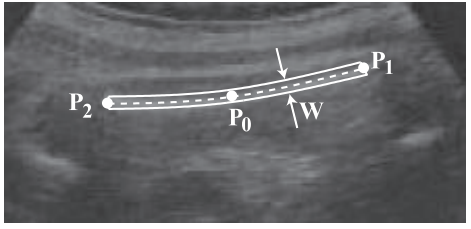

Fig. 1. Parameterization of the femur shape: the axis is a 3 point interpolating spline; the inside region is a $W$-pixels-wide strip around the axis.

given by iterative schemes of the type

$$
\begin{aligned}
& \widehat{\theta}^{(t+1)}=\underset{\theta}{\arg \max }\left\{\log p\left(\mathbf{Z} \mid \theta, \widehat{\phi}^{(t)}\right)\right\} \\
& \widehat{\phi}^{(t+1)}=\underset{\phi}{\arg \max }\left\{\log p\left(\mathbf{Z} \mid \widehat{\theta}^{(t+1)}, \phi\right)\right\},
\end{aligned}
$$

where $\widehat{\theta}^{(t)}$ and $\widehat{\phi}^{(t)}$ are the estimates of $\theta$ and $\phi$ at iteration $t$, respectively (see [7] for convergence properties of this type of algorithm).

\section{IMPLEMENTATION}

We have implemented two contour estimation algorithms: one for the fetal femur, and another for the cranial cross-section. In both cases, the underlying criterion and type of algorithm are those in Equations (4), (5), and (6), although the parameterization of the contour shapes is naturally different.

\subsection{Femur Contour Estimation Algorithm}

Given the form of a fetal femur (usually a straight line segment for a younger fetus, and a slightly curved arc for an older fetus [14]), we adopt an interpolating spline defined by only three points $\theta=\left[P_{0}, P_{1}, P_{2}\right]$. As shown in Fig. 1, the inside region $I(\mathbf{v}(\theta))$ is a $W$-pixels-wide stripe around the spline (dashed line). Although this is a very simple parameterization, it reveals itself rich enough to cover all the possible shapes of fetal femurs.

The algorithm defined by Equations (5) and (6) can be seen as a 2-levels hierarchical scheme of nested algorithms: the inner one (Algorithm 2) updates $\theta$ (Equation (5)), taking $\phi$ fixed. Algorithm 2 is then used by the global algorithm, termed Algorithm $\mathbf{1}$, to solve for both $\theta$ and $\phi$.

\section{Algorithm 1}

Inputs: An initial valid point $\widehat{P}_{0}^{\text {init }}$ (given by the user).

Outputs: The estimates $\widehat{\theta}^{\text {final }}$ and $\widehat{\phi}^{\text {final }}$.

Step 0 (initialization): Set $t=0$ (iteration counter). Set $\widehat{P}_{0}^{(t)}=$ $\widehat{P}_{0}^{\text {init }}$. Set $W$ and initialize $\widehat{P}_{1}^{(t)}$ and $\widehat{P}_{2}^{(t)}$ by maximizing the variance measured in the corresponding inside region, while keeping $\widehat{P}_{0}^{(t)}$ fixed.

Step 1: Given the current contour $\mathbf{v}\left(\widehat{\theta}^{(t)}\right)$, update the estimates of the variance parameter $\phi=\left[\phi_{\text {in }} \phi_{\text {out }}\right]$ according to the ML criterion,

$$
\widehat{\phi}_{\text {in }}^{(t+1)}=\arg \max _{\phi_{\text {in }}}\left\{\log p\left(\mathbf{Z} \mid \widehat{\theta}^{(t)},\left[\phi_{\text {in }} \phi_{\text {out }}\right]\right)\right\}
$$




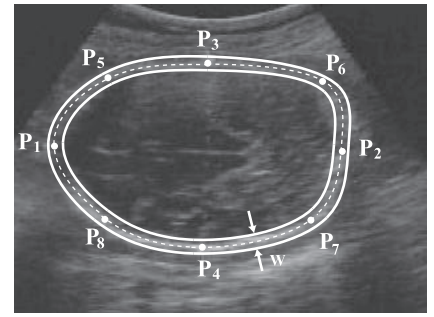

Fig. 2. Parameterization of the cranial cross-section wall.

$$
=\frac{1}{2\left|I^{(t)}\right|} \sum_{(i, j) \in I^{(t)}} Z_{(i, j)}^{2} .
$$

where $I^{(t)}=I\left(\mathbf{v}\left(\widehat{\theta}^{(t)}\right)\right)$ is the current estimate of the inside region, and $\left|I^{(t)}\right|$ denotes the number of pixels in $I^{(t)}$. A similar expression, with $O^{(t)}=O\left(\mathbf{v}\left(\widehat{\theta}^{(t)}\right)\right)$ replacing $I^{(t)}$, is used to obtain $\widehat{\phi}_{\text {out }}^{(t+1)}$.

Step 2: Run Algorithm 2, providing $\widehat{\theta}^{(t)}$ and $\widehat{\phi}^{(t+1)}$ as inputs. Algorithm 2 returns an updated $\widehat{\theta}^{(t+1)}$.

Step 3: If some stopping criterion is met, terminate with outputs $\widehat{\theta}^{\text {final }}=\widehat{\theta}^{(t+1)}$ and $\widehat{\phi}^{\text {final }}=\widehat{\phi}^{(t+1)}$; otherwise, increment $t$ and return to Step 1.

\section{Algorithm 2}

Inputs: A given parameter estimate $\widehat{\phi}$ and an initial contour parameter $\widehat{\theta}$ first

Outputs: An updated contour parameter estimate $\widehat{\theta}^{\text {new }}$.

Step 0 (initialization): Set $q=0$ (iteration counter) and initialize $\widehat{\theta}^{(q)}=\widehat{\theta}^{\text {first }}$.

Step 1: Update the contour parameters according to

$$
\widehat{P}_{0}^{(q+1)}=\underset{P_{0} \in \mathcal{N}\left(\widehat{P}_{0}^{(q)}\right)}{\arg \max } \log p\left(\mathbf{Z} \mid\left[P_{0}, \widehat{P}_{1}^{(q)}, \widehat{P}_{2}^{(q)}\right], \widehat{\phi}\right)
$$

where $\mathcal{N}\left(\widehat{P}_{i}^{(q)}\right)$ is the set of 8 nearest neighbors of $\widehat{P}_{i}^{(q)}$, for $i=0,1,2$. Similar expressions are used for $\widehat{P}_{1}^{(q+1)}$ and $\widehat{P}_{2}^{(q+1)}$

Step 2: If a stopping criterion is met, output $\widehat{\theta}^{\text {new }}=\widehat{\theta}^{(q+1)}$; if not, increment $q$ and go back to Step 1.

\subsection{Head Contour Estimation}

To represent the fetal cranial cross section we have parameterized the contour as a closed 8-point interpolating spline, that is, $\theta=$ $\left[P_{1}, \ldots, P_{8}\right]$. As illustrated in Fig. 2, the inside region $I(\mathbf{v}(\theta))$ is a $W$-pixels-wide stripe around the spline (dashed line).

The estimation algorithm has the same structure as the one described above for the femur. However, head contour estimation turns out to be more difficult, mainly due to the presence of several intracranial structures which can wrongly attract the contour estimate. To avoid these artifacts, we include a new term which forces the contour to move beyond undesired high variance regions. This is similar to a balloon model [4], and it is analogous to providing the contour with an inflating force. The modified estimation criterion is thus

$$
(\hat{\theta}, \hat{\phi})=\arg \max _{\theta, \phi}\{\log p(\mathbf{Z} \mid \theta, \phi)+\lambda b(\theta)\},
$$

where $b(\theta)$ is the balloon term, an increasing function of the contour area; here, we set $b(\theta)$ to the area of the inscribing rectangle. The weight $\lambda$ must be sufficient to allow the contour to move past the undesired regions, but not so strong that it makes the contour move beyond the true cranial wall. We have found experimentally that $\lambda=0.15$ is a good general-purpose choice. We are currently studying ways of adjusting $\lambda$ automatically.

\section{EXPERIMENTS}

\subsection{Synthetic Images}

The first two examples simply illustrate the results of the algorithm using synthetic images generated according to the Rayleigh model. In Fig. 3 we simulate a femur, with the inner and outer variances set to 156 and 116, respectively. The image model parameter estimates obtained were $\widehat{\phi}_{\text {in }}=156.05$ and $\widehat{\phi}_{\text {out }}=115.70$, very close to the true ones. In the example of Fig. 4, resembling a cranial cross-section, the inner and outer regions have variance 60.77 and 42.95 , respectively. The final parameter estimates are $\widehat{\phi}_{\text {in }}=62.49$ and $\widehat{\phi}_{\text {out }}=42.94$.

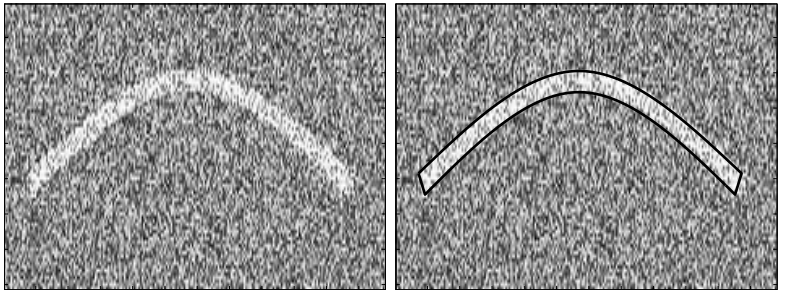

Fig. 3. Synthetic image of a "femur" and the estimated contour.
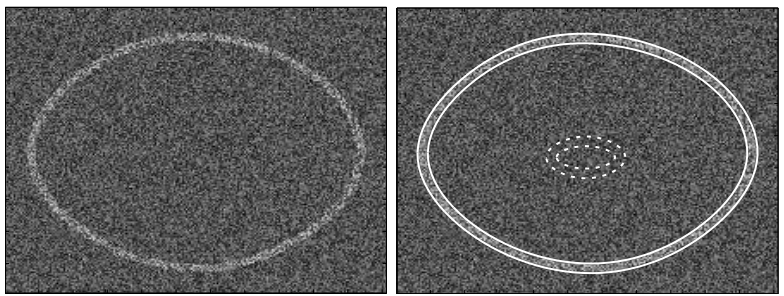

Fig. 4. Synthetic image of a "head" and the estimated contour (the dashed line is the initial contour).

\subsection{Real Images}

Figs. 5 and 6 show examples of femur and cranial cross-section contour estimates on real ultrasound images. Recall that user intervention reduces to indicating a point somewhere on the femur, or inside the head, respectively; no parameter adjustments are needed.

It is hard to quantitatively assess the quality of the results, in the absence of any ground truth. Subjectively, all the estimated contours were considered very good by clinicians. 

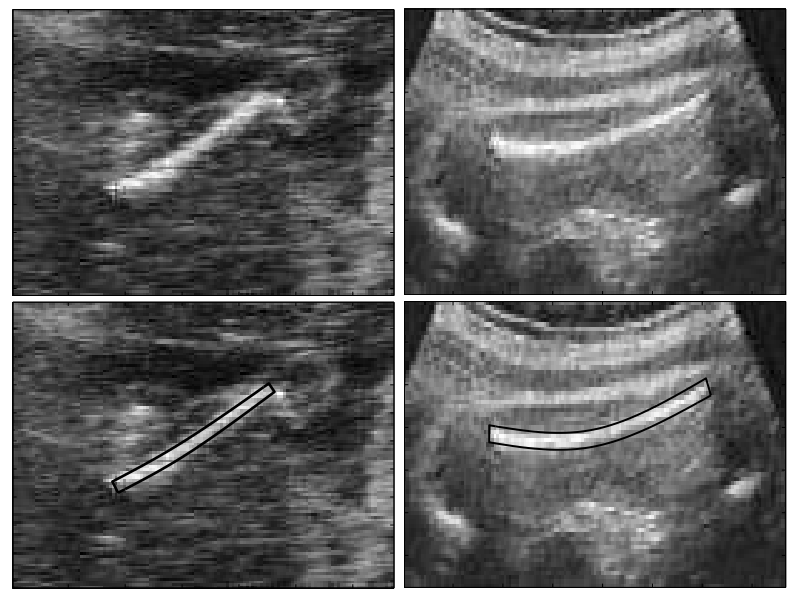

Fig. 5. Examples of femur contour estimation on real images.
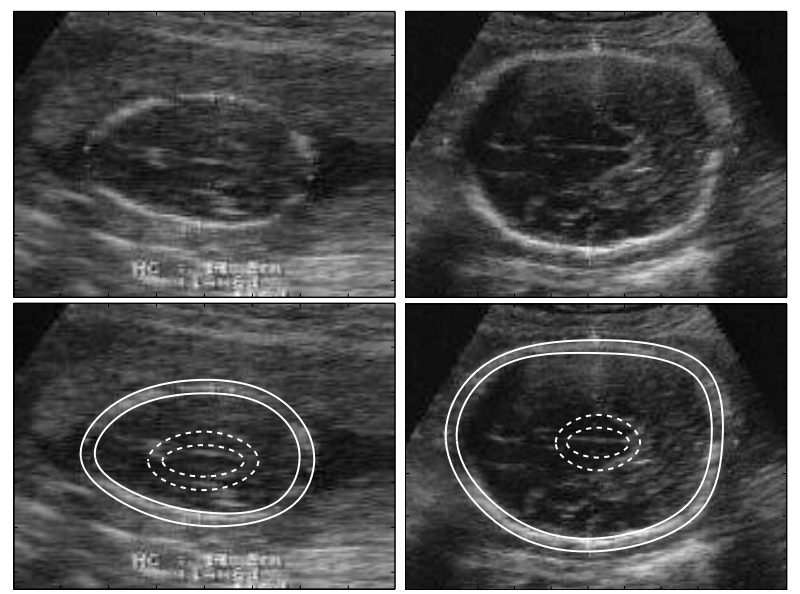

Fig. 6. Examples of cranial cross-section contour estimation on real images.

\subsection{Measurement Consistency}

In order to further evaluate our method, we have tested the implemented algorithms on a group of 30 pairs of images (femur and cranial cross-section of each fetus). On this set of images, manual contours were obtained by trained clinicians, and from these, gestational age (GA) estimates were computed using the FL and BPD parameters (see Section 1). The average difference between the GA estimates obtained from these manual FL and BPD measurements is close to one week. Using automatic contour extraction, the average difference, for this set of images, is 1 day. This suggests a very good consistency of the measurements obtained automatically with the proposed approach.

\section{CONCLUDING REMARKS}

We have described an approach to contour estimation in fetal ultrasound images, based on a maximum likelihood formulation of deformable parametric models. Experiments on synthetic and real images have shown the ability of the proposed method to estimate contours in an unsupervised manner, i.e. adapting to not com- pletely known shapes and completely unknown observation parameters. Preliminary tests suggest that our method leads to more consistent measurements than those based on manual delineation of the structures. A clinical validation study is currently under way.

\section{REFERENCES}

[1] C. Burckhardt, "Speckle in ultrasound b-mode scans," IEEE Trans. on Sonics and Ultrasonics, vol. 25, pp. 1-6, 1978.

[2] A. Chakraborty, L. Staib, and J. Duncan, "Deformable boundary finding influenced by region homogeneity," Proc. CVPR'94, pp. 624-627, 1994.

[3] V. Chalana, T. Winter, D. Cyr, D. Haynor, and Y. Kim, "Automatic fetal head measurements from sonographic images," Academic Radiology, vol. 3, pp. 628-635, 1996.

[4] L. Cohen, "On active contour models and baloons," Computer Vision, Graphics, and Image Processing: Image Understanding, vol. 53, no. 2, pp. 211-218, 1991.

[5] J. Dias and J. Leitão, "Wall position and thickness estimation from squences of echocardiographic images," IEEE Trans. on Medical Imaging, vol. 15, no. 1, pp. 25-38, 1996.

[6] J. Duncan and N. Ayache, "Medical image analysis: Progress over two decades and the challenges ahead," IEEE Trans. on Patt. Anal. and Machine Intell., vol. 22, pp. 85-106, 2000.

[7] M. Figueiredo and J. Leitão, "Bayesian estimation of ventricular contours in angiographic images," IEEE Trans. on Medical Imaging, vol. 11, no. 3, pp. 416-429, 1992.

[8] M. Figueiredo, J. Leitão, and A. K. Jain, "Unsupervised contour representation and estimation using B-splines and a minimum description length criterion," IEEE Trans. on Image Processing, vol. 9, pp. 1075-1087, 2000.

[9] C. Hanna and A. Youssef, "Automated measurements in obstetric ultrasound images," Proc. ICIP'97, pp. 504-507, 1997.

[10] M. Kass, A. Witkin, and D. Terzopolous, "Snakes: Active contour models," Proc. First ICCV, pp. 259-268, 1987.

[11] T. McInerney and D. Terzopoulos, "Topologically adaptable snakes,” Proc. ICCV'95, pp. 840-845, 1995.

[12] T. McInerney and D. Terzopoulos, "Deformable models in medical image analysis: A survey," Medical Image Analysis, vol. 1, pp. 91-108, 1996.

[13] R. Ronfard, "Region-based strategies for active contour models," Int Jour. of Comp. Vis., vol. 13, pp. 229-251, 1994.

[14] R. Sanders and A. James, The Principles and Practice of Ultrasonography in Obstetrics and Gynecology, AppletonCentury-Crofts, Connecticut, 1985.

[15] A. Yuille, D. Cohen, and P. Hallinan, "Feature extraction from faces using deformable templates," in Proc. CVPR'89, 1989, pp. 104-109.

[16] S. Zhu and A. Yuille, "Region competition: Unifying snakes, region growing, energy/Bayes/MDL for multi-band image segmentation," IEEE Trans. on Patt. Anal. and Machine Intell., vol. 18, pp. 884-900, 1996. 